\title{
Reduction of Algebraic Parametric Systems by Rectification of their Affine Expanded Lie Symmetries
}

\author{
Alexandre Sedoglavic \\ ALIEN Project, INRIA Futurs \& LIFL (CNRS, UMR 8022), \\ Université des Sciences et Technologie de Lille, 59655 Villeneuve d'Ascq, France.
}

\begin{abstract}
Lie group theory states that knowledge of a $m$-parameters solvable group of symmetries of a system of ordinary differential equations allows to reduce by $m$ the number of equations. We apply this principle by finding some affine derivations that induces expanded Lie point symmetries of considered system. By rewriting original problem in an invariant coordinates set for these symmetries, we reduce the number of involved parameters. We present an algorithm based on this standpoint whose arithmetic complexity is quasi-polynomial in input's size.
\end{abstract}

\section{Introduction}

Before analysing a biological model described by an algebraic system, it is useful to reduce the number of relevant parameters that determine the dynamics.

Example 1. In order to give an example of such a reduction, let us consider the following Verhulst's logistic growth model with linear predation (see $\S 1.1$ in [1]):

$$
\dot{x}=(a-b x) x-c x, \quad \dot{a}=\dot{b}=\dot{c}=0, \quad \dot{t}=1,
$$

for which all forthcoming computations could be easily performed by hand. Assuming that $a \neq c$, one can represent the flow $(t, x)$ of $(1)$ using parameterization:

$$
t=\mathrm{t} /(a-c), \quad x=(a-c) \mathrm{x} / b,
$$

where $(t, x)$ is the flow of the following differential equation:

$$
\dot{\mathrm{x}}=(1-\mathrm{x}) \mathrm{x} .
$$

In this formulation of (1), parameters $a$ and $c$ were lumped together into $a-c$ and its state variables $x$ and $t$ were nondimensionalise.

Usually, presentation of this kind of simplification relies on rules of thumbs (for example, the knowledge of units in which is expressed the problem when dimensional analysis is used) and thus, there is - up to our knowledge - no complexity results on these kind of reduction methods (see [2] and references therein).

However, these reductions are generally based on the existence of Lie point symmetries of the considered problem (for reduction based on dimensional analysis, see the Theorem 3.22 in [3]). 
Example 1 (continued). The following continuous groups of transformations:

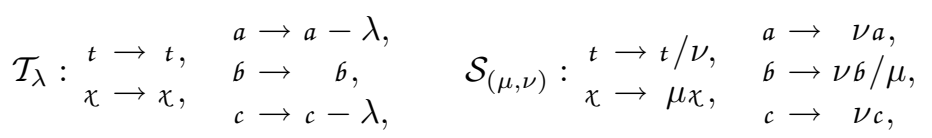

leave invariant system (1) and its solutions. These symmetries are called $e x-$ panded because they act on the expanded space of variables that includes the system parameters in addition to independent and dependent variables.

The system (3) is obtained by 'factoring out' the actions of the symmetries (4) of the original system (1) and thus, it is invariant under these actions. The relations (2) parameterize the solutions of the original system (1) in function of the solution $(\mathrm{t}, \mathrm{x})$ of invariant system (3) and of the free parameters $a, b, c$; they are defined by the composition $\left(\mathcal{S}_{(a / b, a)} \circ \mathcal{T}_{c}\right)(\mathrm{t}, \mathrm{x}, a, b, c)$.

The aim of this note is to show how Lie theory unifies and extends the classical methods (exact lumping, dimensional analysis, etc.) used to simplify algebraic (differential) systems. We adopt a presentation based on algebraic tools closer to the actual computations (mainly Jordan normal form and linear algebra) on which are based our reduction process.

\section{$1.1 \quad$ Related works}

The literature on investigation of the invariants of Lie group action and their applications to algebraic systems is far too vast to be reviewed properly here. The book [4] shows various applications of invariants in the study of dynamical systems under a computer algebra viewpoint. Section 4.1 for example, shows how the knowledge of invariants of a given dynamical system could simplify further computation on it by reducing the degree of involved polynomial expressions. In [5] authors show, given a rational group action, how to compute a complete set of its invariants.

We adopt the same general philosophy - determine some system's symmetries and use their invariants - but our purposes is more to reduce the number of variables involved in these expressions than their degrees (that is for us only a byproduct). Furthermore, while the symmetries considered in $[4,5]$ are quite general - and thus, their computation of invariants are exclusively done using Gröbner bases computations - we restrict ourself to the use of affine Lie symmetries and thus, the required operations are restricted to linear algebra over a number field and univariate polynomial factorization.

\subsection{Main Steps and Tools of the Reduction Process}

The reduction process introduced in this note is based on classical Lie theory but, to the best of our knowledge, it is not described elsewhere in the literature. Let us present now the tools used in this process through our introducing example:

Example 1 (continued). Reduction of Example 1 is done as follow 
Step 1. Determine affine infinitesimal generators that induce expanded Lie symmetries of the considered system. If there is no such derivations, our reduction process stops. In our example, they are:

$$
\delta_{1}:=\frac{\partial}{\partial a}+\frac{\partial}{\partial c}, \quad \delta_{2}:=x \frac{\partial}{\partial x}-b \frac{\partial}{\partial b}, \quad \delta_{3}:=a \frac{\partial}{\partial a}+b \frac{\partial}{\partial b}+c \frac{\partial}{\partial c}-t \frac{\partial}{\partial t} .
$$

The section 3.1 describes the required computations and shows that their complexity is quasi-polynomial in input size.

Step 2. Choose a generator that is a symmetry of the others (above infinitesimal generators form a solvable Lie algebra and our reduction process have to take this property into account). As $\left[\delta_{1}, \delta_{2}\right]=\left[\delta_{2}, \delta_{3}\right]=0$ and $\left[\delta_{1}, \delta_{3}\right]=\delta_{1}$, we could choose $\delta_{1}$ or $\delta_{2}$. Determine a principal element $(\rho:=\log b)$ of this last generator i.e. an element defining a coordinates set in which the derivation $\delta_{2}$ is rectified (equal to the translation $\partial / \partial \rho$ ).

Step 3. The chosen principal element induces an invariantization of the considered system i.e. the system $\dot{\mathrm{x}}=(a-\mathrm{x}) \mathrm{x}-c \mathrm{x}$ that is invariant under the action $\mathcal{S}_{\mu}: \chi \rightarrow \mu \chi, 6 \rightarrow 6 / \mu$ of the one-parameter group of symmetry induced by $\delta_{2}$. The solution $x$ of the original system is then parameterized by $\mathcal{S}_{b}(\mathrm{x})$ where $\mathrm{x}$ is a solution of its invariantization and $b$ is a free parameter.

Repeat Step 1 (supplementary affine symmetries could appear after Step 3).

Let us stress that it is generally hard to find a general infinitesimal generator of a system's symmetry (Step 1) and to give an explicit representation of an invariant coordinates set (Step 2) for it. Thus, we restrict ourself to Lie symmetries associated to affine infinitesimal generators for which invariant coordinates computation is easy (for general case see [5] and references therein). Hence, we do not follows methods developed for general cases because their complexity are likely exponential in input's size while we focus our attention to method of quasi-polynomial complexity.

To conclude, remark that this reduction process works also for purely algebraic system (describing fixed point of a dynamical system for example).

Outline. In the next section, we recall some basic definitions concerning considered systems and related derivations. Then, we present the notion of principal element and show how it could be used in order to define a rectifying coordinates set for general derivations. In the second part of this note, we focus our attention on affine Lie point symmetries in order to propose a probabilistic strategy to compute them and their associated principal elements. We show how previously introduced notions are used in the reduction process by considering invariantization of purely algebraic (resp. differential) system and their parameterization. Finally, in conclusion we make some remarks and suggest possible further works.

\section{Considered systems and associated derivations}

\subsection{Some Algebraic Systems used in Analysis of Biological Model}

Note 2. Notations - Hereafter, we consider an explicit algebraic ordinary differential system $\Sigma$ bearing on $n$ state variables $X:=\left(x_{1}, \ldots, x_{n}\right)$ and depending 
on $\ell$ parameters $\Theta:=\left(\theta_{1}, \ldots, \theta_{\ell}\right)$ :

$$
\Sigma \quad\left\{\begin{array}{l}
\dot{X}=F(t, X, \Theta) \\
\dot{t}=1, \quad \dot{\Theta}=0
\end{array}\right.
$$

Denoting the set $\{1, \ldots, n\}$ by $N$, the letter $\dot{X}$ stands for first order derivatives of state variables $\left(\dot{x}_{\jmath} \mid \jmath \in N\right)$ w.r.t. time $t$ and $F:=\left(f_{\jmath} \mid \jmath \in N\right)$ is a finite subset of $\mathbb{K}(t, X, \Theta)$ where $\mathbb{K}$ is a subfield ( $\mathbb{Q}$ for example) of $\mathbb{C}$. In order to determine the qualitative properties of the dynamical system (6), it is usual to consider the following systems for various subset $J$ of $N$ :

$$
\Sigma_{J} \quad \begin{cases}\dot{x}_{J}=f_{\jmath}(t, X, \Theta), & \forall \jmath \in J, \\ \dot{x}_{\imath}=f_{\imath}(t, X, \Theta)=0, & \forall \imath \in N \backslash J, \\ \dot{t}=1, \quad \dot{\Theta}=0, & \end{cases}
$$

in which some state variables are considered as parameters. In fact, for $\imath$ in $N$, the system $\Sigma_{\{\imath\}}$ defines the so-called $x_{\imath}$-nullcline of $\Sigma$ and the purely algebraic system $\Sigma_{\emptyset}$ defines its fixed points (see examples of applications in [1]).

Remark 3. In the sequel, we are going to avoid - as much as possible - any distinction between time, state variables and parameters i.e. we work in an expanded state space (see [6] for another application of this standpoint); hence, let us denote the set $(t, X, \Theta)$ by $Z:=\left(z_{\imath} \mid 1 \leq \imath \leq 1+\ell+n\right)$ and its cardinal by $m$.

\subsection{Infinitesimal Generators, associated flows and their rectification}

First let us recall some basic facts about derivations.

Definition 4. Given a polynomial algebra $\mathbb{K}[Z]$, a derivation of $\mathbb{K}[Z]$ with constant field $\mathbb{K}$ is an additive mapping $\delta: \mathbb{K}[Z] \rightarrow \mathbb{K}[Z]$ that satisfies Leibniz rules:

$$
\forall\left(\mathrm{f}_{1}, \mathrm{f}_{1}\right) \in \mathbb{K}[Z]^{2}, \quad \delta\left(\mathrm{f}_{1} \mathrm{f}_{2}\right)=\mathrm{f}_{1} \delta \mathrm{f}_{2}+\mathrm{f}_{2} \delta \mathrm{f}_{1},
$$

and have $\mathbb{K}$ in its kernel. We denote by $\operatorname{Der}_{\mathbb{K}} \mathbb{K}[Z]$ the set of all such derivations. The Lie bracket is defined by the $\mathbb{K}$-bilinear map:

$$
\begin{aligned}
{[,]: \operatorname{Der}_{\mathbb{K}} \mathbb{K}[Z] \times \operatorname{Der}_{\mathbb{K}} \mathbb{K}[Z] } & \rightarrow \operatorname{Der}_{\mathbb{K}} \mathbb{K}[Z], \\
\left(\delta_{1}, \delta_{2}\right) & \rightarrow \delta_{1} \delta_{2}-\delta_{2} \delta_{1} .
\end{aligned}
$$

This map is skew-symmetric and satisfies the following Jacobi identity:

$$
\forall\left(\delta_{1}, \delta_{2}, \delta_{3}\right) \subset \operatorname{Der}_{\mathbb{K}} \mathbb{K}[Z], \quad\left[\delta_{1},\left[\delta_{2}, \delta_{3}\right]\right]+\left[\delta_{2},\left[\delta_{3}, \delta_{1}\right]\right]+\left[\delta_{3},\left[\delta_{2}, \delta_{1}\right]\right]=0 .
$$

The set $\operatorname{Der}_{\mathbb{K}} \mathbb{K}[Z]$ is a $\mathbb{K}$ vector-space spanned by the set of canonical derivations $\left\{\partial / \partial z_{1}, \ldots, \partial / \partial z_{m}\right\}$. It is also a Lie algebra with Lie bracket as product.

Remark 5. An algebraic system $\Sigma_{J}$ defined by (7) could be seen as a derivation:

$$
D_{J}:=\frac{\partial}{\partial t}+\sum_{\jmath \in J} f_{\jmath} \frac{\partial}{\partial x_{\jmath}}
$$

associated to the algebraic relations $\left\{f_{\imath}=0, \forall \imath \in N \backslash J\right\}$. 
Derivations considered as infinitesimal generators. The exponentiation of a derivation $\delta$ induces several morphisms as shown by following definitions:

Definition 6. Given a derivation $\delta$ and $\tau$ one of its constant $(\delta \tau=0)$, one can define the exponential map $e^{\tau \delta}:=\sum_{\imath \in \mathbb{N}} \tau^{\imath} \delta^{\imath} / \imath$ ! from $\mathbb{K}[Z]$ into the algebra $\mathbb{K}[[\tau, Z]]$ of power series in the indeterminates $(\tau, Z)$.

1. This map is a morphism that associates to any $\mathrm{f}$ in $\mathbb{K}[Z]$ its Lie series defined by the formal power series $\sum_{\imath \in \mathbb{N}} \tau^{\imath} \delta^{\imath} \mathrm{f} / \imath$ !.

2. The derivation $\delta$ is called the infinitesimal generator of $e^{\tau \delta}$.

3. The formal power series $e^{\tau \delta} Z$ are solutions of the vector field associated to $\delta$. These series form the formal flow of $\delta$; this derivation induces an infinitesimal transformation from $\mathbb{K} \times \mathbb{K}^{m}$ into $\mathbb{K}^{m}$ that associates, under suitable condition of convergence, the evaluation $\left(e^{\tau \delta} Z\right)(\mathcal{V})$ to any parameter $\tau$ in $\mathbb{K}$ and any initial point $\mathcal{V}$ in $\mathbb{K}^{m}$; this map is the action of the flow $e^{\tau \delta}$ on $\mathbb{K}^{m}$.

Example \%. Hence, the $\mathbb{C}(x)$-morphism $\sigma_{\tau}: x \rightarrow e^{\tau} x$ could be defined by the exponential map $\sigma_{\tau}:=e^{\tau \delta}$ where $\delta$ denotes the derivation $x \partial / \partial x$ acting on the field $\mathbb{C}(x)$. The set $\left\{\sigma_{\tau} \mid \tau \in \mathbb{C}\right\}$ is a one-parameter group of automorphisms.

Lemma 8. Given two derivations $\partial$ and $\delta$, the Baker Campbell Hausdorff formula states that the relation $e^{\partial} e^{\delta}=e^{\delta} e^{\partial} e^{[\partial, \delta]}$ holds.

The next section presents some how some derivations could be expressed as translation in a suitable coordinates set.

\section{Some Algebraic Tools for Rectification of an Infinitesimal Generator.}

Principal element. Forthcoming manipulations are based on the existence of a special element that behaves as a time variable for considered derivation as shown by the following definition:

Definition 9. An $\rho$ element in a algebra $\mathrm{A}$ is principal for a derivation $\delta$ acting on $\mathrm{A}$ if the relation $\delta \rho=1$ holds.

In order to determine a principal element $\rho$ of a derivation $\delta$, one have to solve the following partial differential equation $\delta \rho=1$. As this is not a trivial task and as not every derivation has such an element, we are going in the sequel to restrict our manipulation to the following kind of principal elements:

Lemma 10. Given a derivation $\delta$ of $\mathbb{K}[Z]$, if there exists an element $\varrho$ in $\mathbb{K}[Z]$,

1. such that the relations $\delta \varrho \neq 0$ and $\delta^{2} \varrho=0$ hold, then the fraction $\rho:=\varrho / \delta \varrho$

2. and a constant $\lambda$ of $\delta$ such that the relation $\delta \varrho=\lambda \varrho$ holds, then for any constant $c$ of this derivation, the transcendental element $\rho:=\log (c \varrho) / \lambda$

is a principal element $\rho$ of $\delta$. The element $\varrho$ is called the preprincipal element of $\delta$ associated to $\rho$. 
Remark 11. As we adopt an algebraic standpoint in this note, this lemma requires to consider in the sequel the localization $\mathbb{K}[\rho, Z]_{(\wp)}$ of $\mathbb{K}[\rho, Z]$ at the multiplicative closed set $\wp:=\left\{(\delta \varrho)^{\imath} \mid \imath \in \mathbb{N}\right\}$ (resp. $\left.\wp:=\left\{\varrho^{\imath} \mid \imath \in \mathbb{N}\right\}\right)$. In fact, given any canonical derivation $\partial / \partial z$ of $\mathbb{K}[Z]$, there exists one, and only one, canonical derivation of $\mathbb{K}[\rho, Z]_{(\wp)}$ extending $\partial / \partial z$ and such that the following usual relations $\partial \rho / \partial z=\partial \varrho / \partial z-\left(\rho /(\delta \varrho)^{2}\right) \partial \delta \varrho / \partial z($ resp. $\partial \rho / \partial z=(1 / \varrho) \partial \varrho / \partial z)$ are well defined in $\mathbb{K}[\rho, Z]_{(\wp)}$ (for the sake of simplicity, we use the same notation for derivations acting on $\mathbb{K}[Z]$ and their extension to derivation acting on $\left.\mathbb{K}[\rho, Z]_{(\wp)}\right)$. This shows that the Lie algebra $\operatorname{Der}_{\mathbb{K}} \mathbb{K}[\rho, Z]_{(\wp)}$ is well defined.

Example 12. For any element $h$ in $\mathbb{K}(Z)$, we consider the $\log$ arithm $\log h$ i.e a transcendental field extension $\mathbb{K}(Z, \log h)$ and the associated derivation extension such that $\delta \log h=\delta h / h$. Hence, the derivation $\delta:=x \partial / \partial x$ acting on $\mathbb{C}(x)$ has a unique extension to a derivation $\bar{\delta}$ acting on $\mathbb{C}(x, \log (x))$ such that the relation $\bar{\delta} \log (x)=1$ holds.

Construction of a Rectifying Coordinate Ring. Principal elements of a derivation $\delta$ allow to construct a rectifying field in which $\delta$ acts as a simple translation.

Lemma 13. Given a derivation $\delta$ and one of its principal element $\rho$, let us define the following formal operator:

$$
\pi_{\delta, \rho}:=\sum_{\imath \in \mathbb{N}}(-\rho)^{\imath} \frac{\delta^{\imath}}{\imath !} .
$$

As $\delta$ is a derivation, this operator induces a homomorphism and the following exact sequence:

$$
0 \rightarrow \operatorname{ker} \pi_{\delta, \rho} \rightarrow \mathbb{K}[\rho, Z]_{(\wp)} \stackrel{\pi_{\delta, \rho}}{\longrightarrow} \mathbb{K}[[\rho, Z]]_{(\wp)} \rightarrow \mathbb{K}[\zeta] \rightarrow 0
$$

where the variables set $\zeta$ denotes the set $\pi_{\delta, \rho} Z$ of formal power series.

Remark 14. To prove that the map $\pi_{\delta, \rho}$ is a homomorphism, one can use the same argument then whose used in the proof stating the same property for the exponential map $e^{\delta}$. By construction $\pi_{\delta, \rho} \rho$ is equal to 0 . Thus, the kernel ker $\pi_{\delta, \rho}$ contains the ideal $\rho \mathbb{K}[\rho, Z]_{(\wp)}$ and is not trivial (see also Proposition 30).

Using exact sequence (13), we could define a coordinate ring $\mathbb{K}[\zeta]$ that is isomorphic to the quotient algebra $\mathbb{K}[\rho, Z]_{(\wp)} /\left(\operatorname{ker} \pi_{\delta, \rho}\right) \mathbb{K}[\rho, Z]_{(\wp)}$ and a rectifying ring $\mathbb{K}[\zeta, \rho]$ that is its finitely generated extension. To explain this terminology, first remark that the derivation $\delta$ acting on $\mathbb{K}[\rho, Z]_{(\wp)}$ could be easily extended to a derivation acting on $\mathbb{K}[[\rho, Z]]_{(\wp)}$ and thus to $\mathbb{K}[\rho, \zeta]$. The following lemma states that the derivation $\delta$ is rectified when we consider its action on $\mathbb{K}[\rho, \zeta]$ :

Lemma 15. With previously introduced notations, the following relations hold:

$$
\delta \rho=1, \quad \delta \zeta=0 .
$$


Sketch of proof. The first relation is the definition of a principal element. Elements $\zeta$ are defined by the series $\sum_{\imath \in \mathbb{N}}(-\rho)^{\imath} \delta^{\imath} Z / \imath$ !. By Leibniz' rule, we have:

$$
\forall \imath \in \mathbb{N}, \quad \delta\left((-\rho)^{\imath} \frac{\delta^{\imath}}{\imath !}\right)=(-1)^{i}\left(\rho^{\imath} \frac{\delta^{2+1}}{\imath !}+\rho^{\imath-1} \frac{\delta^{\imath}}{(\imath-1) !}\right),
$$

and thus, derivation's linearity proves the last relations.

The morphism $\pi_{\delta, \rho}$ induces a coordinates change allowing to express the derivation $\delta$ as a simple translation $\partial / \partial \rho$ in this new coordinates set. We do not describe further this coordinates change, because we are just going to use some of its properties and not its exact formulation. Forthcoming considerations are based on the fact that the relations $\delta \zeta=0$ imply that the relations $e^{\tau \delta} \zeta=\zeta$ hold. Thus, the morphism $\pi_{\delta, \rho}$ maps the coordinate ring $\mathbb{K}[Z]$ of the ambient space $\mathbb{K}^{m}$ onto a coordinate ring invariant under the action of the flow $e^{\tau \delta}$.

\subsection{Expanded Lie Point Symmetries and their Determining System}

Let us define now the derivations used in the sequel.

Definition 16. Given a derivation $\delta$, an algebraic system $\Sigma_{J}$ and the associated derivation $D_{J}, \delta$ is an infinitesimal generator of an expanded Lie point symmetry of $\Sigma_{J}$ if there exists a constant $\lambda$ of $D_{J}$ such that the following relations hold:

$$
\begin{array}{cc}
D_{J} \delta(t)=\frac{\partial \delta(t)}{\partial t}+\sum_{\jmath \in J} f_{\jmath} \frac{\partial \delta(t)}{\partial x_{\jmath}}=-\lambda, & \\
\sum_{z \in Z} \delta(z) \frac{\partial f_{\imath}}{\partial z}-\frac{\partial \delta\left(x_{\imath}\right)}{\partial t}-\sum_{\jmath \in J} f_{\jmath} \frac{\partial \delta\left(x_{\imath}\right)}{\partial x_{\jmath}}=\lambda f_{\imath}, \quad & \forall \imath \in J, \\
\delta f_{\imath}=\sum_{z \in Z} \delta(z) \frac{\partial f_{\imath}}{\partial z}=\lambda f_{\imath}, \quad \forall \imath \in N \backslash J, \\
D_{J} \delta(\theta)=\frac{\partial \delta(\theta)}{\partial t}+\sum_{\jmath \in J} f_{\jmath} \frac{\partial \delta(\theta)}{\partial x_{\jmath}}=0, \quad \forall \theta \in \Theta .
\end{array}
$$

These relations form the determining system of $\Sigma_{J}$ expanded Lie point symmetries.

Remark 17. Solution space structure - Derivations $\delta$ satisfying (16)-(19) form a Lie sub-algebra of $\operatorname{Der}_{\mathbb{A}} \mathbb{K}[Z]$ denoted by $\operatorname{LieSym}(\Sigma)$. Furthermore, if $J_{1}$ is a subset of $J_{2}$, the Lie algebra $\operatorname{LieSym}\left(\Sigma_{J_{1}}\right)$ is a sub-algebra of $\operatorname{LieSym}\left(\Sigma_{J_{2}}\right)$.

Remark 18. Considered Lie symmetries vs general Lie symmetries - The definition 16 is designed for our algebraic purposes but is only a restriction of the general definition of Lie point symmetries (see [3]). In fact, remark that if the considered algebraic system $\Sigma_{J}$ is 
- a vector field $(J=N)$, this definition reduces to the classical one of Lie point symmetries based on the Lie bracket i.e. $[D, \delta]=\lambda D$;

- a purely algebraic system $(J=\emptyset)$, this definition is more restrictive than the classical definition presented in Section 2.1 of [3]. In fact, let us consider the following system $f_{1}:=x_{1}^{2}+y_{1}^{2}-1, f_{2}:=x_{2}^{2}+y_{2}^{2}-1, f_{3}:=x_{2} y_{1}-y_{2} x_{1}$ and the derivation $\delta:=x_{2} \partial / \partial y_{1}-y_{2} \partial / \partial x_{1}+x_{1} \partial / \partial y_{2}-y_{1} \partial / \partial x_{2}$. The relations $\delta f_{1}=2 f_{3}, \delta f_{2}=-2 f_{3}, \delta f_{3}=f_{1}-f_{2}$ hold i.e. the derivation $\delta$ leaves invariant the ideal spanned by $f_{1}, f_{2}, f_{3}$ and thus according to [3], $\delta$ is the infinitesimal generator of a one-parameter group of Lie symmetry (a family of morphism $e^{\tau \delta}$ parameterized by a constant $\tau$ ) that leaves the ideal spanned by $\left\{f_{1}, f_{2}, f_{3}\right\}$ invariant but not each of these polynomials (as shown by the relation $\left.e^{\tau \delta} f_{1}=f_{1} \cos ^{2} \tau+f_{2} \sin ^{2} \tau+2 f_{3} \cos \tau \sin \tau\right)$. This kind of derivations are not taken into account in this note. This restriction is motivated by computational purposes i.e. the general definition of a Lie point symmetry for an algebraic system $F=0$ implies that we could - at least-determine 0 in the quotient algebra $\mathbb{K}[Z] / F \mathbb{K}[Z]$; as this task is not in the complexity class considered in this note, we made a first restriction to the set of Lie symmetry used in our work by only considering solution of system (16)-(19).

Furthermore, there is little hope to solve the general partial differential problem (16) - (19); thus, we restrict our solution space to affine infinitesimal generators for which the associated determining equations form a linear system.

\section{Affine derivation and associated invariantization}

Definition 19. Let us denote by $\operatorname{AffDer}_{\mathbb{K}} \mathbb{K}[Z]$ the following set of derivations:

$$
\left\{\delta=\sum_{z \in Z} \delta(z) \frac{\partial}{\partial z} \mid \delta\left(z_{1}\right):=b_{z_{1}}+\sum_{z_{2} \in Z} a_{z_{1} z_{2}} z_{2},\left(b_{z_{1}}, a_{z_{1} z_{2}} \mid z_{2} \in Z\right) \in \mathbb{K}^{m+1}\right\} .
$$

Note 20. Notation - Given a derivation $\delta$ in $\operatorname{AffDer}_{\mathbb{K}} \mathbb{K}[Z]$, we are going in the sequel to consider $Z$ as a vector and use the following matricial notations:

$$
\mathcal{A}_{\delta}=\left(a_{z_{1} z_{2}}\right)_{\left(z_{1}, z_{2}\right) \in Z^{2}}, \quad \mathcal{B}_{\delta}=\left(b_{z}\right)_{z \in Z}, \quad \delta Z=\mathcal{A}_{\delta} Z+\mathcal{B}_{\delta}
$$




\subsection{Determining System defining Affine Infinitesimal generators}

Lemma 21. For an affine infinitesimal generator $\delta$ in $\operatorname{AffDer}_{\mathbb{K}} \mathbb{K}[Z]$, the associated determining system (16) - (19) reduces to the following linear system:

$$
\left(\begin{array}{ccc}
0 & \cdots & 0 \\
\frac{\partial f_{1}}{\partial z_{1}} & \cdots & \frac{\partial f_{1}}{\partial z_{m}} \\
\vdots & & \vdots \\
\frac{\partial f_{n}}{\partial z_{m}} & \cdots & \frac{\partial f_{n}}{\partial z_{m}} \\
0 & \cdots & 0 \\
\vdots & & \vdots \\
0 & \cdots & 0
\end{array}\right)\left(\mathcal{A}_{\delta} Z+\mathcal{B}_{\delta}\right)-\mathcal{A}_{\delta}\left(\begin{array}{c}
1 \\
f_{1} \mathbb{1}_{1 \in J} \\
\vdots \\
f_{n} \mathbb{1}_{n \in J} \\
0 \\
\vdots \\
0
\end{array}\right)=\lambda\left(\begin{array}{c}
1 \\
f_{1} \\
\vdots \\
f_{n} \\
0 \\
\vdots \\
0
\end{array}\right),
$$

where $\mathbb{1}_{\imath \in J}$ is equal to 1 if the index $\imath$ is in $J$ and 0 otherwise.

Remark 22. Probabilistic resolution of determining system defining affine derivation - The system (22) could be rewritten in a the more convenient matricial notation $M(Z) K=0$ where $M(Z)$ is a $m \times(m+1) m$ matrix with coefficients in $\mathbb{K}[Z]$ and $K$ is a vector whose $(m+1) m$ coefficients are the coefficients of $\mathcal{A}_{\delta}$ and $\mathcal{B}_{\delta}$. Affine derivations that are solution of this determining system, are given by the kernel of $M(Z)$ in a field $\mathbb{K}$. Kernel computation could be done by the following probabilistic method. Indeterminates $Z$ are specialized in matrix $M(Z)$ to some random value in $\mathbb{K}^{m}$ in order to obtain a matrix $M_{1}$ over the field $\mathbb{K}$; the resulting linear system $M_{1} K$ could be underdetermined and thus, several specializations should be considered in order to obtain a linear system $L_{\imath}$ defined by $M_{1} K=\cdots=M_{\imath} K=0$. The rank $r_{\imath}$ of $L_{\imath}$ increases with $\imath$ and the specialization process could be stopped when $r_{\jmath}=r_{\jmath+1}$; the considered system $L_{\jmath}$ could then be solved using a numerical method. The specialization set for which this process fails to find a correct solution is a zero-dimensional algebraic variety and thus, its probability of failure is low.

However, there is an infinite way to choose a basis of the kernel computed above. But, one can use Lenstra, Lenstra and Lovász' basis reduction algorithm in order to obtain a reduced basis in the sense that less variables are involved in each infinitesimal generators definition.

To conclude, remark that some solutions of system (22) are spurious for our purposes since they describe the same flow and should be discarded. In fact, consider the problem $\Sigma$ defined by $\dot{x}=\theta x$ : the base field of $\operatorname{LieSym}(\Sigma)$ is the constant field of the derivation $D:=\partial / \partial t+\theta x \partial / \partial x$; thus, as $\partial / \partial t$ is in $\operatorname{LieSym}(\Sigma)$ and $\theta$ is a constant of $D, \theta \partial / \partial t$ is another infinitesimal generators representing the same Lie symmetry then $\partial / \partial t$. These two derivations define the same Lie symmetry but are given by two different solutions of system (22).

\subsection{Principal Element Computation for Affine Derivation}

Lemma 23. Given a derivation $\delta$ in $\operatorname{AffDer}_{\mathbb{K}} \mathbb{K}[Z]$. If there exits a vector of $\delta$ 's constants denoted by $C:=\left(c_{1}, \ldots, c_{m}\right)$ such that the relations 
1. ${ }^{t} C \mathcal{A}_{\delta}=0$ and ${ }^{t} C \mathcal{B}_{\delta} \neq 0$ hold, then the fraction ${ }^{t} C Z /{ }^{t} C\left(\mathcal{A}_{\delta} Z+\mathcal{B}_{\delta}\right)$

2. ${ }^{t} \mathcal{A}_{\delta} C=\lambda C$ and $\delta \lambda=0$ hold, then the element $\left(\log { }^{t} C\left(Z+\mathcal{B}_{\delta} / \lambda\right)\right) / \lambda$

is a principal element of $\delta$.

Sketch of proof. 1) Consider the polynomial ${ }^{t} C Z$ denoted by $\varrho$. Using notation (21), remark that $\delta \varrho$ is equal to the linear combination ${ }^{t} C\left(\mathcal{A}_{\delta} Z+\mathcal{B}_{\delta}\right)$. Thus, the conditions on $C$ given in the first item show that $\delta \varrho$ is a constant different of 0 and thus $\delta^{2} \varrho$ is equal to 0 . The first assertion of Lemma 10 is sufficient to conclude in that case. 2) Consider the polynomial ${ }^{t} C\left(Z+\mathcal{B}_{\delta} / \lambda\right)$ denoted by $\varrho$. With the hypothesis of the second item, the element $\delta \varrho$ is equal to $\lambda \varrho$ and thus, the second assertion of lemma 10 is satisfied and the transcendental element $\log (\varrho) / \lambda$ is a principal element of $\delta$.

Remark 24. Computational strategy - This lemma shows that in order to find a principal element for a derivation $\delta$ in $\operatorname{AffDer}_{\mathbb{K}} \mathbb{K}[Z]$, one have first to check condition 1) and if it is not satisfied, one have to find an eigenvector of $\mathcal{A}_{\delta}$.

\subsection{Flow of a Affine Derivation and Resulting Quotient Space}

Finding a coordinates change required to place a given derivation in rectified form is essentially the same problem as solving it in the first place. This could be easily done for affine derivation using the Jordan normal form as shown bellow.

Remark 25. Jordan normal form - Given a $m \times m$-matrix $\mathcal{A}_{\delta}$ associated to a derivation $\delta$, if its minimal polynomial $p(\xi)$ is $\prod_{\imath=1}^{w} p_{\imath}$ with $p_{\imath}=\left(\xi-\lambda_{\imath}\right)^{\alpha_{\imath}}$ and $\sum_{\imath=1}^{w} \alpha_{\imath}=m$, then there exists a change of coordinates $\mathcal{P}$ such that:

$$
\mathcal{A}_{\delta}=\mathcal{P}\left(\begin{array}{cccc}
J_{1} & 0 & \cdots & 0 \\
0 & J_{2} & \ddots & \vdots \\
\vdots & \ddots & \ddots & 0 \\
0 & \cdots & 0 & J_{w}
\end{array}\right) \mathcal{P}^{-1}, \text { with } J_{\imath}:=\lambda_{\imath} \operatorname{Id}_{\alpha_{\imath} \times \alpha_{\imath}}+\left(\begin{array}{ccccc}
0 & 1 & 0 & \cdots & 0 \\
0 & 0 & 1 & \ddots & \vdots \\
\vdots & \ddots & \ddots & 0 \\
0 & \cdots & 0 & 1 \\
0 & \cdots & 0 & 0
\end{array}\right)
$$

if $\lambda_{\imath}$ is different from 0 and $J_{\imath}:=\lambda_{\imath} \operatorname{Id}_{\alpha_{\imath} \times \alpha_{\imath}}$ otherwise; the symbol $\operatorname{Id}_{\alpha_{\imath} \times \alpha_{\imath}}$ denotes the identity $\alpha_{\imath} \times \alpha_{\imath}$-matrix. This canonical form and Lemma 23 allow to compute preprincipal and associated principal element for any affine derivation $\delta$.

Hypotheses 1. From now, we suppose that the base field $\mathbb{K}$ is $\mathbb{C}$ in order to contain all eigenvalues of the matrix $\mathcal{A}_{\delta}$ and to define the quantities related to principal elements (exponentials and logarithms).

Given an affine derivation $\delta$, one of its preprincipal element $\varrho$ and the associated principal element $\rho$, let us interpret geometrically the manipulation done in section 2.2. 
Flow associated to a derivation and induced equivalence classes. To do so and following Definition 6-3, we consider the application defined by the linear system of ordinary differential equations associated to our affine derivation $\delta$ :

$$
\begin{aligned}
\Psi: \mathbb{K} \times \mathbb{K}^{m} & \rightarrow \mathbb{K}^{m}, \\
(\tau, \mathcal{W}) & \rightarrow\left(e^{\tau \delta} Z\right)(\mathcal{W})=\exp \left(\tau \mathcal{A}_{\delta}\right) \mathcal{W}+\int_{0}^{\tau} \exp \left((\tau-s) \mathcal{A}_{\delta}\right) \mathcal{B}_{\delta} \mathrm{d} s
\end{aligned}
$$

this application could be computed numerically or using the following relations:

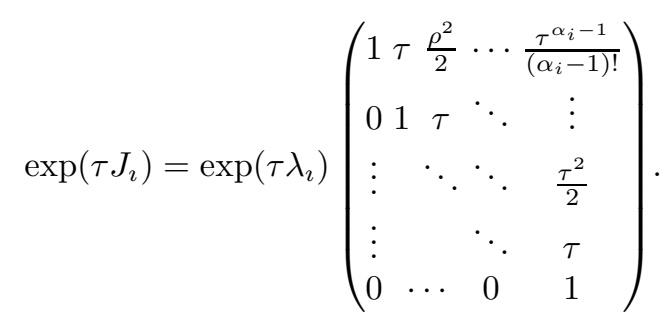

Note 26. Orbits of the flow - The image of $\mathbb{K} \times \mathcal{W}$ by $\Psi$ constitutes an orbit of the flow $e^{\tau \delta}$. This standpoint induces an equivalence relation $\sim$ among the point of $\mathbb{K}^{m}$, with $\mathcal{V}_{1}$ being equivalent to $\mathcal{V}_{2}$ if these points lie in the same orbit of $\Psi$. Let us denotes by $\mathcal{H}_{\delta}$ the set of equivalence classes a.k.a. the set of orbits. In the sequel, we suppose for the sake of conciseness that all the orbits have the same dimension i.e. we implicitly exclude from our statements the lower-dimensional orbits associated to the variety in $\mathbb{K}^{m}$ defined by the ideal $\{\delta(z)=0 \mid z \in Z\}$.

All forthcoming manipulations rely on the following remark:

Remark 2\%. Invariantization - Any object (algebraic relations, derivations, etc.) that is invariant under the action of the flow $e^{\tau \delta}$ will have a counterpart on the lower-dimensional variety $\mathcal{H}_{\delta}$ whose representation - the invariantization of the considered object - completely characterize the original object.

Note 28. As a first illustration, let us remark that any function $\mathrm{f}: \mathbb{K}^{m} \rightarrow \mathbb{K}$ invariant for the flow $e^{\tau \delta}$ is invariant along its orbits and therefore there is a welldefined induced function $\tilde{\mathrm{f}}: \mathcal{H}_{\delta} \rightarrow \mathbb{K}$; conversely, given a function $\tilde{\mathrm{f}}: \mathcal{H}_{\delta} \rightarrow \mathbb{K}$, there is an invariant function $\mathrm{f}: \mathbb{K}^{m} \rightarrow \mathbb{K}$ defined by the relation $\mathrm{f}(\mathcal{V}):=\tilde{\mathrm{f}}(h)$ if $\mathcal{V}$ is in the orbit $h$. Hence, we obtain the following result:

Lemma 29. There is a one-to-one correspondence between (polynomial) functions on $\mathbb{K}^{m}$ invariant under the action of the flow $e^{\tau \delta}$ and arbitrary (polynomial) functions on $\mathcal{H}_{\delta}$.

In order to represent $\mathcal{H}_{\delta}$, one can first find an algebraic representation of the orbits of the flow and then use a cross section of these orbits (see [5] and references therein for more details). This general approach is based on Gröbner basis computation and treats general problems that exceed the scope of this note. Instead, we are going to use Lemmas 13 and 29 in order to give an algebraic description of $\mathcal{H}_{\delta}$ whose computation is based mainly on Jordan decomposition. 
Algebraic Representation of $\mathcal{H}_{\delta}$. Consider the formal operator $\pi_{\delta, \rho}$ introduced in Lemma 13. Lemma 15 implies that the image of $\pi_{\delta, \rho}$ is invariant under the action the flow $e^{\tau \delta}$. Thus, by describing the kernel of $\pi_{\delta, \rho}$, we obtain an algebraic description of functions on $\mathbb{K}^{m}$ invariant under the action of the flow $e^{\tau \delta}$. Lemma 29 shows that this description induces an algebraic representation of $\mathcal{H}_{\delta}$. The following lemma recapitulates these points when $\delta$ is an affine derivation:

Proposition 30. Given an affine derivation $\delta$, one of its preprincipal element $\varrho$ and the associated principal element $\rho$, one can define a homomorphism $\pi_{\delta, \rho}$ on an algebra of constants $\mathbb{K}[\zeta]$ of $\delta$ using the formal operator (12) as follow:

$$
\begin{aligned}
0 \rightarrow q_{\rho} \mathbb{K}[Z]_{(\wp)} & \rightarrow \mathbb{K}[Z]_{(\wp)} \stackrel{\pi_{\delta, \rho}}{\longrightarrow} \mathbb{K}[\zeta] \rightarrow 0 \\
\mathrm{p}(Z) & \longrightarrow \mathrm{p}(\zeta)
\end{aligned}
$$

where $\zeta$ is equal to $\pi_{\delta, \rho} Z$ and $q_{\rho}$ is equal to ${ }^{t} C Z$ if the preprincipal element $\varrho$ is defined by the case 1) in Lemma 23 and to ${ }^{t} C\left(Z+\mathcal{B}_{\delta} / \lambda\right)-1$ otherwise. The set of equivalence classes $\mathcal{H}_{\delta}$ could be identify with the hyperplane $V\left(q_{\rho}\right)$ of dimension $m-1$ defined in $\mathbb{K}^{m}$ by the linear form $q_{\rho}$. Furthermore,

1. the map $\pi_{\delta, \rho}$ induces a projection that associates to any point $\mathcal{V}$ in $\mathbb{K}^{m}$ s.t. $\wp(\mathcal{V}) \neq 0$, the point $\left(\pi_{\delta, \rho} Z\right)(\mathcal{V}):=\sum_{\imath \in \mathbb{N}}\left((-\rho(Z))^{\imath}\left(\delta^{\imath} Z\right) / \imath !\right)(\mathcal{V})$ of $V\left(q_{\rho}\right)$.

2. the points composing an orbit of $e^{\tau \delta}$ are projected to a single point in $V\left(q_{\rho}\right)$.

3. the orbit of $e^{\tau \delta}$ passing through a point in $V\left(q_{\rho}\right)$ is projected on this point.

Sketch of proof. Consider a principal element $\rho$ of $\delta$ and its defining preprincipal element $\varrho$ such that $\delta \varrho=\mu \varrho\left(\right.$ resp. $\delta \varrho \neq 0$ and $\left.\delta^{2} \varrho=0\right)$. Then, the relation $\pi_{\delta, \rho} \varrho=\varrho e^{-\log (\varrho)}\left(\operatorname{resp} . \pi_{\delta, \rho} \varrho=0\right)$ holds and thus $\pi_{\delta, \rho} \varrho$ is equal to 1 (resp. 0 ) (to be more precise $\partial \pi_{\delta, \rho} \varrho / \partial z$ is equal to 0 for all $z$ in $Z$ and thus $\pi_{\delta, \rho} \varrho$ is in $\mathbb{K}$ ). If we denotes $\varrho-1$ (resp. $\varrho$ ) by $q_{\rho}$, the ideal $q_{\rho} \mathbb{K}[Z]_{(\wp)}$ is include in ker $\pi_{\delta, \rho}$. Furthermore, as we suppose that the flow $e^{\tau \delta}$ acts regularly, its orbits have the same dimension 1 and thus, the associated invariant coordinates ring $\mathbb{K}[\zeta]$ is of dimension $m-1$. Hence, the quotient algebra $\mathbb{K}[Z]_{(\wp)} / q_{\rho} \mathbb{K}[Z]_{(\wp)}$ allows to describe algebraically $\mathbb{K}[\zeta]$. Let us now, see some properties of the associated algebraic variety $V\left(q_{\rho}\right)$. The first above assertion is just the definition of a projection on $V\left(q_{\rho}\right)$.

2) Remark that as the flow $e^{\tau \delta}$ is an homomorphism and relation $e^{\tau \delta} \delta=\delta e^{\tau \delta}$ holds, we have $\rho\left(e^{\tau \delta} Z\right)^{\imath}\left(\delta^{2} e^{\tau \delta} Z\right)=\rho\left(e^{\tau \delta} Z\right)^{\imath}\left(e^{\tau \delta} \delta^{2} Z\right)=e^{\tau \delta}\left(\rho(Z)^{2} \delta^{\imath} Z\right)$, for all integer $\imath$. In order to show that the relation $\rho\left(e^{\tau \delta} Z\right)=e^{\tau \delta} \rho(Z)$ holds, remark that as $e^{\tau \delta}$ is a homomorphism, $\varrho\left(e^{\tau \delta} Z\right)$ is equal to $e^{\tau \delta} \varrho(Z)$. If the principal element $\rho$ is equal to $\log (\varrho) / \lambda($ resp. $\varrho / \delta \varrho)$ with $\delta \varrho=\lambda \varrho\left(\right.$ resp. $\delta \varrho \neq 0$ and $\left.\delta^{2} \varrho=0\right)$ then the transcendental element $\log \left(e^{\tau \delta} \varrho\right) / \lambda\left(\right.$ resp. $\left.e^{\tau \delta}(\varrho / \delta \varrho)\right)$ is equal to $\log \left(\varrho e^{\tau \lambda}\right) / \lambda$ (resp. $(\varrho+\tau \delta \varrho) / \delta(\varrho+\tau))$ and thus, $\rho\left(e^{\tau \delta} Z\right)$ is equal to $\rho+\tau$ which is also equal to $e^{\tau \delta} \rho$. Above relations show that $\left(\pi_{\delta, \rho} e^{\tau \delta} Z\right)(\mathcal{V})$ is equal to $\left(e^{\tau \delta} \pi_{\delta, \rho} Z\right)(\mathcal{V})$. As the flow leaves the image of $\pi_{\delta, \rho}$ invariant (i.e. $e^{\tau \delta} \pi_{\delta, \rho} Z=\pi_{\delta, \rho} Z$ ), this quantity is equal to $\left(\pi_{\delta, \rho} Z\right)(\mathcal{V})$. Hence, two points $\mathcal{V}$ and $\left(e^{\tau \delta} Z\right)(\mathcal{V})$ in the same orbit of $e^{\tau \delta}$ are projected onto the same point of $V\left(q_{\rho}\right)$.

3) If $\mathcal{W}$ is in $V\left(q_{\rho}\right)$ then $q_{\rho}(\mathcal{W})$ is equal to 0 and thus, $\rho(\mathcal{W})$ is also equal to 0. In that case, by construction $\left(\pi_{\delta, \rho} Z\right)(\mathcal{W})$ is equal to $\mathcal{W}$. 
The orbits of $e^{\tau \delta}$ cross the hyperplane $V\left(q_{\rho}\right)$ transversally. In the sequel, we denote this hyperplane by $\mathcal{H}_{\delta}$. Let us show now how works our reduction process.

\subsection{Invariantization and Parameterization}

Reduction of Algebraic Systems. Consider the variety $V(F)$ in $\mathbb{K}^{m}$ defined by the ideal spanned by $F$ in $\mathbb{K}[Z]_{(\wp)}$ (for the sake of simplicity, we suppose that $F$ is prime).

Parameterization of an algebraic variety invariant under the action of $e^{\tau \delta}$. If $\delta$ is an affine derivation such that the relation $\delta F=\lambda F$ holds, it is an infinitesimal generator of a Lie symmetry $e^{\tau \delta}$ that leaves the variety $V(F)$ invariant as shown by the following relations holding for all $\mathcal{W}$ in $\mathbb{K}^{m}$,

$$
\mathcal{V}:=\left(e^{\tau \delta} Z\right)(\mathcal{W}), F(\mathcal{V}):=\left(F\left(e^{\tau \delta} Z\right)\right)(\mathcal{W})=\left(e^{\tau \delta} F(Z)\right)(\mathcal{W})=e^{\tau \lambda} F(\mathcal{W})
$$

As shown by Proposition 30, the hyperplane $\mathcal{H}_{\delta}$ is a linear cross-section of the orbit of $e^{\tau \delta}$ i.e. a variety that intersects these orbits in a single point. Furthermore, there is a variety $W$ induced by $V(F)$ in $\mathcal{H}_{\delta}$ such that the variety $V(F)$ is the image of $\mathbb{K} \times W$ by the action of the flow $e^{\tau \delta}$. Let us described now $W$.

Invariantization of purely algebraic systems. The variety $W$ is defined by the intersection $V\left(F \bmod q_{\rho}\right) \cap \mathcal{H}_{\delta}\left(\right.$ if $q_{\rho}(\mathcal{W})$ and $\left(F \bmod q_{\rho}\right)(\mathcal{W})$ are equal to zero, then the relations $F(\mathcal{W})=0$ hold). As $q_{\rho}$ is linear, a description of $W$ is obtained by a simple substitution in the equations describing $V(F)$ (compare with the replacement invariant studied in [5]) as shown by the following example:

Example 31. Let us consider the following purely algebraic system:

$$
\Sigma: \quad(y-b)^{2}+a^{2}=l^{2} / 4, \quad(x-a)^{2}+b^{2}=l^{2} / 4, \quad x^{2}+y^{2}=l^{2} .
$$

Using results of Section 3.2, we determine its expanded affine Lie symmetries:

$$
\delta_{1}:=x \frac{\partial}{\partial x}+y \frac{\partial}{\partial y}+a \frac{\partial}{\partial a}+b \frac{\partial}{\partial b}+l \frac{\partial}{\partial l}, \delta_{2}:=-y \frac{\partial}{\partial x}+x \frac{\partial}{\partial y}+(b-y) \frac{\partial}{\partial a}+(x-a) \frac{\partial}{\partial b} .
$$

As $l$ is a preprincipal element of $\delta_{1}$, the solutions $Z$ of system (28) are represented by the parameterization $Z=e^{\tau \delta_{1}} \mathrm{Z}_{1}$ where $\mathrm{Z}_{1}$ are the solutions of an invariant system obtained by the intersection of $(28)$ with the hyperplane $l=1$ :

$$
\forall z \in\{x, y, a, b, l\}, \begin{gathered}
z=e^{\tau} \mathrm{z}_{1}, \\
\mathrm{l}_{1}=1,
\end{gathered} \quad\left\{\begin{array}{c}
\left(\mathrm{y}_{1}-\mathrm{b}_{1}\right)^{2}+\mathrm{a}_{1}^{2}=1 / 4, \\
\left(\mathrm{x}_{1}-\mathrm{a}_{1}\right)^{2}+\mathrm{b}_{1}^{2}=1 / 4, \\
\mathrm{x}_{1}^{2}+\mathrm{y}_{1}^{2}=1 .
\end{array}\right.
$$

As $\delta_{1}$ and $\delta_{2}$ form an abelian Lie algebra, this last derivation is an infinitesimal generators of a Lie symmetry of $\delta_{1}$; it could be used to reduce further the system (30) (the Lie algebra spanned by $\delta_{1}$ and $\delta_{2}$ is abelian and thus solvable). In fact, the linear form $a-I b-x$ is a preprincipal element of $\delta_{2}$ associated to the 
principal element $\log (a-I b-x) / I$. Using symmetry $\delta_{2}$, solutions of system (30) could be represented as follow:

$$
\forall z_{1} \in\left\{\mathrm{x}_{1}, \mathrm{y}_{1}, \mathrm{a}_{1}, \mathrm{~b}_{1}\right\}, \begin{aligned}
& \mathrm{z}_{1}=e^{\tau \delta \mathrm{z}_{2}} \mathrm{z}_{2}, \\
& \mathrm{x}_{2}=\mathrm{a}_{2}-I \mathrm{~b}_{2}-1,
\end{aligned} \quad\left\{\begin{array}{c}
\left(\mathrm{y}_{2}-\mathrm{b}_{2}\right)^{2}+\mathrm{a}_{2}{ }^{2}=1 / 4 \\
\left(1+I \mathrm{~b}_{2}\right)^{2}+\mathrm{b}_{2}{ }^{2}=1 / 4, \\
\left(\mathrm{a}_{2}-I \mathrm{~b}_{2}-1\right)^{2}+\mathrm{y}_{2}{ }^{2}=1 .
\end{array}\right.
$$

In this particular example, the positive dimensional system (28) is represented

- by a zero-dimensional algebraic system (31) that furnishes initial values $\mathrm{Z}_{2}$

- to an explicit linear differential system whose solutions are $Z_{1}=e^{\tau \delta_{2}} Z_{2}$ (this system associated to the derivation $\delta_{2}$ is simple enough to be explicitly solved in closed form but in more complicated cases it could also be considered as a black box representation solved by purely numerical methods);

- these values $Z_{1}$ constitute an initial condition set of the linear differential system - induced by the derivation $\delta_{1}$-such that resulting solutions $Z$ parameterize the variety defined by system (31).

We show now that the same type of results exists for differential systems.

\section{Reduction of Differential systems.}

Hypotheses 2. Restriction on Symmetries Specific to Differential Case - Lie symmetries of a given vector field $D$ acting only on its state variables $(\dot{z} \neq 0)$ could be used for Lie based integration but not for the previous reduction process. Thus, we suppose that $D$ have an expanded Lie symmetry that acts at least on one of its parameter $(D z=0)$ and that there is an associated principal elements such that the associated linear form $q_{\rho}$ satisfies the relation $D q_{\rho}=0$.

Invariantization of an infinitesimal generator. Given any derivation $D$ acting on $\mathbb{K}[Z]$, the sequence (26) induces a derivation $\bar{D}$ acting on $\mathbb{K}[\zeta]$ such that the relation $\pi_{\delta, \rho} \circ D=\bar{D} \circ \pi_{\delta, \rho}$ holds. The exponentiation of $\bar{D}$ (see Definitions 6) induces a flow $e^{\tau \bar{D}}$ on the hyperplane $\mathcal{H}_{\delta}$ that is the invariantization of the flow $e^{\tau D}$ acting on $\mathbb{K}^{m}$. Under above hypotheses, the flow $e^{\tau \bar{D}}$ is just the restriction of $e^{\tau D}$ on $\mathcal{H}_{\delta}$; in fact, as $D q_{\rho}=0$ the relation $e^{\tau D} q_{\rho}=q_{\rho}$ holds and thus, the flow $e^{\tau D}$ maps any point of $\mathcal{H}_{\delta}$ to another point of this hyperplane. The set of orbits of $e^{\tau D}$ in $\mathbb{K}^{m}$ is projected onto the set of orbits of $e^{\tau D}$ in $\mathcal{H}_{\delta}$. Let us see now the condition on $\delta$ and $D$ that allows to parameterize the set of orbits of $e^{\tau D}$ in $\mathbb{K}^{m}$ by the set of orbits of $e^{\tau D}$ in $\mathcal{H}_{\delta}$ and the map $e^{\tau \delta}$.

Parameterization of vector field $D$ invariant under the action of the flow $e^{\tau \delta}$. If $\delta$ is the infinitesimal generator of a symmetry of derivation $D$, according to Definition 16, the relation $[D, \delta]=\lambda D$ holds. The Baker Campbell Hausdorff formula (Lemma 8) shows that the relation $e^{\tau_{1} \delta} e^{\left(1+\tau_{1} \lambda\right) \tau_{2} D} e^{-\tau_{1} \delta}=e^{\tau_{2} D}$ holds. This implies that any orbit of $e^{\tau D}$ in $\mathbb{K}^{m}$ is the image of an orbit of $e^{\tau D}$ in $\mathcal{H}_{\delta}$ by the flow $e^{\tau \delta}$. Let us explicit all the process described above through an example. 
Example 32. Consider a FitzHugh Nagumo model (see $\S 7$ in [1]):

$$
\dot{a}=\dot{b}=\dot{c}=\dot{d}=0, \quad \dot{x}=\left(x-x^{3} / 3-y+d\right) c, \quad \dot{y} / \mathrm{d} t=(x+a-b y) / c .
$$

The derivation $\delta:=\partial / \partial y+b \partial / \partial a+\partial / \partial d$ is an infinitesimal generator of the following one-parameter group $y \rightarrow y+\lambda, a \rightarrow a+b \lambda, d \rightarrow d+\lambda$ that is composed of symmetries of the system (32). As the relation $\delta d=1$ holds, $d$ is a (pre)principal element of $\delta$ and the solutions $Z:=\{x, y, a, b, c, d\}$ of system (32) are described by the parameterizations $Z=e^{d \delta} \mathrm{Z}$ where $\mathrm{Z}$ are solutions of a differential system on the hyperplane $V(d)$; hence, $Z$ are given by the equations:

$$
y=\mathrm{y}+d, a=\mathrm{a}-b d, \quad \dot{x}=\left(x-x^{3} / 3-\mathrm{y}\right) c, \dot{\mathrm{y}}=(x+\mathrm{a}-b \mathrm{y}) / c .
$$

\section{Conclusion}

In this note, we consider the computation of affine expanded Lie symmetries of a given algebraic system and show how this system could be rewrite in an invariant coordinates set for these symmetries in order to reduce the number of involved parameters. As this process is based on the computation of Jordan normal form and numerical linear algebra, its complexity is quasi-polynomial in input's size and likely polynomial for the great majority of practical cases.

Extension of the reduction process to more general types of derivations. The manipulation presented in previous sections for affine derivations could be used for non-affine symmetries that occurs in practice as shown below.

Example 33. Let us consider the following algebraic system:

$$
1-x+x^{2} y=0, \quad b-x^{2} y=0 .
$$

One can check that the following infinitesimal generator

$$
\delta:=x^{2} \partial / \partial x+(1-2 x y) \partial / \partial y+x^{2} \partial / \partial b
$$

is associated to the following one-parameter group of automorphisms:

$$
e^{\lambda \delta}: x \rightarrow \frac{x}{1-x \lambda}, \quad y \rightarrow\left(y+\frac{\lambda}{1-x \lambda}\right)(x \lambda-1)^{2}, \quad b \rightarrow b+x \frac{x \lambda}{1-x \lambda},
$$

that is a one-parameter group of Lie point symmetries of the system (34). Remark that $\rho:=b /(x(x-b))$ is a principal element of $(35)(\delta \rho=1)$ and thus, all that we have done previously could be repeated i.e. the invariant coordinate set:

$$
\pi_{\delta, \rho} x=x-b, \quad \pi_{\delta, \rho} y=\left(y x^{2}-b\right) /(b-x)^{2}, \quad \pi_{\delta, \rho} b=0,
$$

allows to represent the solutions set $(x, y)$ of $(34)$ as follow

$$
x=\mathrm{x}+b, y=\left(\mathrm{yx}^{2}+b\right) /(\mathrm{x}+b)^{2} \quad \forall(\mathrm{x}, \mathrm{y}) \text { s.t. } 1-\mathrm{x}+\mathrm{x}^{2} \mathrm{y}=0, \mathrm{x}^{2} \mathrm{y}=0 .
$$

The results presented here could likely be extended for more general types of derivations but we do not know if the associated computations are feasible. 
Acknowledgments. The author is grateful to G. Renault, É. Schost, and M. Safey El Din for many pleasant and useful discussions related to this note.

\section{References}

1. Murray, J.D.: Mathematical Biology. Volume 17 of Interdisciplinary Applied Mathematics. Springer (2002)

2. Khanin, R.: Dimensional Analysis in Computer Algebra. In Mourrain, B., ed.: Proceedings of the 2001 International Symposium on Symbolic and Algebraic Computation, London, Ontario, Canada, ACM, ACM press (2001) 201-208

3. Olver, P.J.: Applications of Lie groups to differential equations. 2 edn. Volume 107 of Graduate Texts in Mathematics. Springer Verlag (1993)

4. Gatermann, K.: Computer algebra methods for equivariant dynamical systems. Number 1728 in Lecture Notes in Mathematics. Springer New York (2000)

5. Hubert, E., Kogan, I.: Rational invariants of an algebraic group action. Construction and rewriting. Journal of Symbolic Computation (2006) (to appear).

6. Burde, G.I.: Expanded Lie group transformations and similarity reductions of differential equations. In Nikitin, A.G., Boyko, V.M., Popovych, R.O., eds.: Symmetry in nonlinear mathematical physics Part I. Volume 43 of Proceedings of Institute of Mathematics of NAS of Ukraine., Kiev, Ukraine (2002) 93-101 